\title{
"Fito-hormônios": ciência e natureza no tratamento do climatério
}

| ${ }^{1}$ Ivone Manzali de Sá I

Resumo: Um novo produto chamado "fitohormônio" foi elaborado para substituir a terapia de reposição hormonal (TRH) nos últimos anos. Este desenvolvimento está associado a dois contextos históricos, a saber: o anúncio dos riscos associados à terapia de reposição hormonal (TRH) para mulheres no climatério no ano de 2002, e uma mudança de percepção do público consumidor e laboratórios farmacêuticos a respeito das plantas medicinais. Algumas plantas já utilizadas por comunidades tradicionais para sintomas associados ao climatério foram pesquisadas cientificamente nas últimas décadas e, como resultado, uma nova categoria de medicamento foi constituída. A partir das teorias ator-rede e de translação, de Bruno Latour, analisa-se a construção desta categoria de medicamento, e como a ideia de "natural" foi ressignificada pelos atores envolvidos na rede sócio-técnica.

> Palavras-chave: climatério; TRH; plantas medicinais; natureza.

\author{
${ }^{1}$ Mestrado em Ciências \\ Biológicas (Botânica), \\ Etnobotânica, pelo Museu \\ Nacional (UFRJ); doutoranda \\ em História das Ciências \\ da Saúde na COC-Flocruz; \\ pesquisadora colaboradora do \\ Museu Nacional (UFRJ) e visitor \\ scholar na University of Hawaii \\ of Manoa. Endereço eletrônico: \\ ivmanzali@gmail.com
}


No final da década de 1990 e início de 2000, chegou ao Brasil, através de mídia farmacêutica especializada, a notícia de uma alternativa "natural" para a terapia de reposição hormonal (TRH) de origem sintética, até então largamente utilizada pelas mulheres ocidentais para aliviar os sintomas do climatério. Apesar de o climatério ser um fenômeno fisiológico - expresso pela diminuição dos níveis dos hormônios estrogênio e progesterona nas mulheres, levando à interrupção da menstruação e possíveis sintomas associados como ondas de calor, secura vaginal e depressão -, sua medicalização vem ocorrendo desde as últimas décadas do século XX através da TRH. Tal terapêutica faz parte de um longo e importante processo de construção da ideia de um corpo feminino, a partir da descoberta, síntese e comercialização de hormônios sexuais desde o início do século XX, como tem sido amplamente discutido, em trabalhos de autoras que atuam no campo de estudos de gênero e ciência, especialmente na produção de Oudshoorn (1993, 1990) e Rohden (2008).

A alternativa para a terapêutica de reposição hormonal ficou conhecida como "fito-hormônios", isto é, substâncias de origem vegetal com características hormônios-like. ${ }^{2}$ Este trabalho analisa como se constituiu essa nova categoria de medicamentos para o climatério e como a ideia de "natureza" e seu correlato "natural" é ressignificada pelo discurso médico-científico e pelos demais atores que compõem a rede sócio-técnica, que resulta na produção desses tipos de hormônios. Do ponto de vista metodológico, as fontes são artigos e livros científicos relativos à mídia especializada, bem como artigos de jornais, revistas e sites da internet para o público leigo e propagandas de produtos.

No que se refere à perspectiva analítica, tomamos por base a teoria atorrede e o conceito de translação descrita por Bruno Latour (1983, 1994, 1997). Esse autor discute os processos de formatação da sociedade a partir de um fato científico, produzidos em ambiente de laboratório, em pequena escala, e sua translação para ambientes macrossociais, através de uma mudança de escala (do micro para o macro). A visibilidade de substâncias ou micro-organismos antes não identificados, mas que no laboratório se tornam visíveis através de inscrições, ${ }^{3}$ possibilitaria este processo.

No que se refere especificamente à produção e difusão dos fito-hormônios, verificamos que uma das estratégias de divulgação e convencimento do médico ao 
prescrevê-los foi o recrutamento de ginecologistas, ligados a centros universitários

de referência (formadores de opinião), publicação de artigos e, especialmente, o patrocínio de eventos científicos como cursos, simpósios, congressos, etc. O papel crucial deste ator na rede foi apontado por Rohden (2008) e Oudshoorn (1990, 1993), que identificaram um campo em que se faz presente a atuação de três grupos em especial: os cientistas de laboratório, a indústria farmacêutica e os médicos ginecologistas.

Na década de 1960, o estrogênio e a progesterona sintéticos eram indicados para controlar distúrbios nos sintomas do climatério, distúrbios menstruais e tratamento da infertilidade, mas tornaram-se populares e indicados para todas as mulheres a partir do período da menopausa, por influência do livro de divulgação científica Feminine Forever (1966), do médico ginecologista americano Robert Wilson (VIGETA, 2004). Nesse livro, o autor faz apologia ao direito da mulher se manter feminina e sexualmente ativa após a menopausa, utilizando preventivamente a TRH. Suas ideias se propagaram no meio médico e leigo, levando milhares de mulheres a utilizarem esta terapêutica, provocando um deslizamento da TRH para a categoria discutida por Azize (2006) como "medicamentos de estilo de vida", em que o objetivo de uso do medicamento estaria associado ao bem-estar do consumidor e não a um estado patológico, necessariamente.

Analisamos a construção desta nova categoria de medicamentos e a identificação dos atores envolvidos, acompanhando a trajetória das plantas medicinais utilizadas tradicionalmente no controle dos sintomas do climatério. ${ }^{4}$ Observamos, então, como as plantas medicinais se transformam em medicamentos, depois de submetidas a processos de laboratórios e por intermédio de sua circulação na mídia leiga e especializada, num contexto sócio-cultural-econômico específico. Seguindo as linhas desta trajetória, outra dimensão analítica é que a ideia de natureza é modulada conceitualmente pelos diferentes atores que participam dessa rede sócio-técnica.

\section{A terapia de reposição hormonal em questão: natureza, ciência e mercado}

No ano de 1975, foram publicados dois artigos no periódico The New England Journal of Medicine, mostrando uma ligação entre a terapia de reposição com estrogênio em mulheres no climatério e o aumento do risco de câncer de 
endométrio (ZIEL; FINKLE, 1975; SMITH et al., 1975). Outros artigos, nos anos subsequentes, mostraram que o câncer do endométrio poderia estar associado à terapia de reposição hormonal, em função dos níveis de estrogênio (LIPSETT, 1977; WEISS, 1978; KHANDEKAR et al., 1978; WEISS et al., 1979). Desta forma, tal medicação passou a ser indicada somente para mulheres histerectomizadas, enquanto que para as mulheres na fase do climatério passouse a conjugar estrogênio com progesterona. Esta nova terapêutica foi então preconizada como protetora de doenças cardíacas, e continuou sendo indicada como preventiva em mulheres menopausadas.

Menos de 20 anos depois, a TRH foi colocada em questão pela Women's Health Initiative (WHI) - promovida, em 1991, pelo National Institute of Health (NIH) do governo dos Estados Unidos, que tinha entre outros objetivos o acompanhamento pela investigação médica, para verificar a adequação de protocolos nos casos de problemas cardiovasculares, câncer e osteosporose. Em uma de suas pesquisas, o WHI alertava para os riscos de doença arterial coronariana, acidente vascular cerebral, assim como trombose e câncer de mama associados à $\mathrm{TRH}$, afirmando que os riscos eram, em muitos casos, superiores aos benefícios de alívio dos sintomas relacionados ao climatério (WHI, 2009). A divulgação dos resultados desta pesquisa no ano 2000 provocou forte reação da comunidade médica, gerando trabalhos sobre procedimentos da clínica médica, medicalização, e validade de outros recursos terapêuticos em substituição à TRH, principalmente plantas medicinais e alimentícias utilizadas por diferentes sistemas médicos tradicionais. A partir da publicação e difusão da pesquisa do WHI, reportagens questionaram a pesquisa em medicina. Artigos intitulados: "Postmenopausal hormone replacement therapy: How could we have been so wrong?” (LAINE, 2002), "TRH x WHI: a estrela vai a nocaute" (GAMA et al., 2004), entre outros, foram divulgados na mídia especializada e leiga, tanto norte-americana como brasileira, colocando em questão alguns paradigmas da pesquisa científica.

Coincidentemente, em 10 de julho de 2002, o jornal The New York Times publicou um artigo das jornalistas Kolata e Petersen (2002), revelando que Dr. Robert Wilson, autor do livro Feminine Forever, recebera financiamento do Laboratório Farmacêutico Wyeth-Ayerst para a publicação do livro e também para manutenção da organização Wilson Research Foundation em Nova York. O laboratório Wyeth-Ayerst era o fabricante do hormônio estrogênio, chamado 
Premarin $^{\circledR}$, que somente no ano de 1975 esteve entre os cinco fármacos mais prescritos nos Estados Unidos (KOLATA; PETERSEN, 2002). Essa reportagem, somada ao impacto da divulgação dos resultados da pesquisa da TRH pela WHI, trouxe desconforto à comunidade médica e opinião pública, expresso em artigos da área médica, como os já citados no texto, além de matérias divulgadas com destaque em vários jornais e revistas acompanhados pela Rede Feminista de Saúde (OBSERVATÓRIO DA IMPRENSA, 2002). Um exemplo foi a matéria de capa da revista Época publicada em julho de 2002 (VEIGA; SEGATTO, 2002), intitulada "Traídas pela medicina: Um golpe na confiança", que reificava o clima de insegurança e desconfiança dos médicos e mulheres consumidoras de hormônios sintéticos na terapia de reposição hormonal.

Curiosamente, os balcões de farmácias e consultórios médicos foram demandados por uma "alternativa natural" aos hormônios sintéticos, que pudesse ao mesmo tempo ser segura e eficaz contra os sintomas da menopausa (IFTODA et al., 2006), como afirmam as médicas Vieira e Navarro no artigo "Síndrome Climatérica": "após a divulgação dos dados do WHI, houve muito interesse e procura pelos 'hormônios naturais', com a falácia de que por serem naturais, estes não ofereceriam qualquer risco à saúde da mulher" (VIEIRA; NAVARRO, 2007, p. 106).

\section{A natureza ressignificada}

É importante ressaltar que tal evento se dá no contexto norte-americano fortemente influenciado pelo movimento New Age e da contracultura dos anos 1960/70, que propagava uma visão de mundo intimamente articulada aos valores da liberdade sexual e da experiência sensível, expressando nesta direção o interesse e busca por práticas terapêuticas ligadas à natureza e a antigos sistemas médicos, como a medicina tradicional chinesa (MTC), ayurveda, xamanismo, entre outros, em geral "reinterpretadas e reapropriadas culturalmente de acordo com padrôes ocidentais" (LUZ, 2007, p. 60).

Também podemos, por outro lado, entender este movimento sob a perspectiva de Campbell (1997), que identifica que estaria ocorrendo no Ocidente, desde o século XVIII, "um processo de 'orientalização"s caracterizado pelo deslocamento da teodiceia ${ }^{6}$ tradicional por outra que é essencialmente oriental na sua natureza" (CAMPBELL, 1997, p. 5). Para esse autor, os anos 1960 foram expressivos 
na emergência de valores associados ao romantismo como uma reação ao establishment, identificados como pertencentes à sociedade moderna. $\mathrm{O}$ autor vincula este processo ao que ele chama de "ética romântica", que já teria contornos nítidos na revolução industrial, influenciando o consumo moderno (CAMPBELL, 2005). Neste movimento, que segundo Duarte (2004, 2005), podemos chamar de neo-romântico, as ideias de uma ligação espiritual entre o ser humano e a natureza, o holismo e consequentemente a ecologia, entre outras, se popularizaram e se tornaram ambíguas, agregando valores da modernidade. ${ }^{7}$

Autores como Lenoble (1990) e Strathern (1992), a partir de campos de trabalhos diversos, defendem o fato de que a ideia de natureza esteve desde sempre presente no mundo ocidental, permeando vários momentos históricos e intelectuais. Lenoble (1990) considera que a ideia de uma natureza "salvadora", "sábia", "original" é anterior a Rousseau, sendo observada na sociedade ocidental, tanto quanto em qualquer outra civilização, "como um sintoma".

[...] a esperança de que as verdadeiras soluçōes e a verdadeira razão serão encontradas renunciando às ideias recebidas: os nossos esforços revelam-se infrutíferos, mas, para lá de nossos esforços, a verdadeira razão manteve-se intacta no homem mais simples, o homem da Natureza. Afastamo-nos da razão ao querer tudo bem demais, o remédio encontrar-se-á num regresso à Natureza (LENOBLE, 1990, p. 235-6).

Já Strathern (1992), ao tomar como objeto o tema do parentesco britânico, busca demonstrar a existência concomitante de "mudanças" e "continuidades" nos sentidos conferidos ao conceito de natureza, demonstrando como esse conceito pode ser modificado pelas alterações impressas pela cultura sem, contudo, romper necessariamente com o seu valor intrínseco. Duarte, por sua vez, considera que "a categoria natureza é crucial para a ordenação dos horizontes modernos, sob diferentes roupagens e através de diferentes tradições de conhecimento" (DUARTE et al., 2005, p. 22).

Corroborando as considerações desses autores sobre a presença e reinterpretação da ideia de natureza ao longo da história da sociedade ocidental e mesmo na atualidade, podemos observar neste estudo de caso, a presença de uma diversidade de sentidos dessa ideia, muitas vezes articulada de forma contraditória até mesmo dentro do mesmo domínio de conhecimento, como as ciências médicas. É nestes termos que podemos considerar que todo um mercado e público consumidor das chamadas "medicinas alternativas" ou "terapias naturais" floresceu em alguns países do mundo ocidental sob uma orientação que privilegiava uma abordagem 
holística do ser humano, como a utilização de produtos naturais, muitas vezes artesanais, os quais podem ser entendidos como expressão desse processo mais amplo de ressignificação do sentido do mundo da "natureza".

A análise mercadológica das empresas e indústrias de produtos naturais, especialmente de medicamentos derivados de plantas em 1998, baseada na pesquisa de Jörg Grunwald, observa aumento significativo, passando de uma posição marginal para se integrar ao mainstream do mercado biomédico. $\mathrm{Na}$ Europa, entre 1987 e 1993, o mercado de fitoterápicos cresceu com taxas mais elevadas que o mercado farmacêutico na sua totalidade (FERREIRA, 1998). Este movimento da sociedade ficou conhecido como "onda verde". Tal tendência mercadológica levou ao ingresso de novos atores nessa rede, quando a partir dos anos 1990, grandes laboratórios farmacêuticos incorporaram pequenas indústrias de medicamentos fitoterápicos ou abriram novas linhas de produtos com tais características (FERREIRA, 1998).

\section{Do mundo da natureza aos laboratórios: a rede sócio- técnica dos fito-hormônios}

Neste cenário, no final dos anos 1990, novos produtos foram apresentados aos públicos leigo e especializado para substituir a TRH. Eles passaram a utilizar plantas medicinais como matérias-primas - Angelica sinensis (Oliv.) Diels, Cimicifuga racemosa (L.) Nutt., Glycine max (L.) Merr. (soja) e Dioscorea villosa L. (cará ou inhame), entre outras espécies - e sua divulgação era dirigida a um público específico (NEWALL et al., 1996; ALONSO, 1998; SCHULZ et al., 2002). Alguns grupos de médicos e pacientes buscavam medicamentos "naturais", mas que tivessem sido submetidos ao processo técnico-científico para atender aos critérios de qualidade e segurança segundo as especificações de controle de qualidade dos produtos fabricados em grandes laboratórios farmacêuticos (GAEDCKE; STEINHOFF, 2003; CAPASSO et al., 2003; WAGNER; WISENAUER, 2006). Desta forma, as plantas medicinais utilizadas no tratamento do climatério em seu estado in natura - na forma de drogas vegetais secas rasuradas para serem administradas como chás, percebidas como produtos naturais que fomentaram o mercado das medicinas alternativas - não seriam neste momento suficientemente "científicas" para atender aos novos critérios de médicos e pacientes. Além de atender a tais critérios, o novo produto deveria 
ao mesmo tempo contemplar o modelo de precisão de "doses regulares" da biomedicina, sintetizando assim a cadeia de produção que envolvia os processos de purificação, quantificação e padronização de um novo medicamento (WAGNER; WISENAUER, 2006).

Através de parcerias firmadas entre empresas farmacêuticas e laboratórios de pesquisas, um novo preparado vegetal foi desenvolvido, agregando características específicas. Ele deveria ser originário de uma planta medicinal com conhecimento tradicional agregado; manter as características principais da planta de origem; e atender às expectativas do paradigma mecanicista da biomedicina de "doses regulares”, como um balizador de segurança e eficácia (GALENA, 2012a, 2012b). Esse preparado, denominado "extrato seco padronizado", foi resultado de purificações da planta, que teoricamente deveria conter as substâncias originais da espécie vegetal, porém melhoradas em sua performance químico-biológica, além de ter substâncias químicas de referência, com quantidades regulares, permitindo assim ao clínico prescrever o medicamento segundo estas substâncias de referência, e não necessariamente pelas características da planta de origem. A quantificação e a padronização passaram a ser atributos fundamentais nesta nova abordagem das plantas medicinais, atendendo a um só tempo os critérios de controle de qualidade de uma indústria farmacêutica e ao paradigma da biomedicina de doses regulares.

No processo de desenvolvimento do extrato seco padronizado, redes de pesquisa foram articuladas em diversas áreas de conhecimento, envolvendo profissionais com formação em etno-farmacologia, agronomia, botânica, fitoquímica, farmacologia e medicina (SONAGLIO et al., 2003). No entanto, a comunicação entre os atores da rede, em geral, foi feita através da publicação dos artigos nos periódicos científicos e eventos das diferentes áreas de conhecimento, ou até mesmo por artigos de difusão científica. Tal comunicação, entretanto, tem maior alcance entre os pares que fazem parte de um mesmo campo, pois geralmente periódicos de uma área de conhecimento específico não são lidos por pesquisadores de outras áreas. Sendo assim, parece que neste momento do processo, os integrantes da rede sócio-técnica não se articulam de maneira tão intensa.

A aplicação dos métodos científicos na pesquisa deste novo produto pressupôs alguns desafios, que passaram pela ideia de padronização da matéria-prima do ponto de vista qualitativo e quantitativo. Buscava-se uma espécie vegetal (padrão 
botânico), que deveria conter teores de determinadas substâncias (padrão

químico) para garantir o resultado esperado, isto é, aquele que foi testado nos ensaios biológicos de laboratório (pré-clínicos e clínicos). No mundo do laboratório científico, diversidade e variabilidade, valores intrínsecos ao produto "natural" não são bem-vindos.

Uma vez que o produto foi desenvolvido, outros dois importantes atores foram acionados para serem incorporados à rede: o médico ginecologista e a paciente. Os médicos especializados em ginecologia tiveram papel de destaque no acesso e monopólio do saber sobre o corpo feminino. Por outro lado, historicamente as mulheres já estavam socializadas nessa rede, a partir do processo de produção de medicamentos advindos da pesquisa hormonal, e na construção da necessidade de medicalização durante o período do climatério feminino (ROHDEN, 2008). Para melhor entender a participação do médico ginecologista como um agente da prescrição do fito-hormônio, é importante entender este ator, do ponto de vista histórico, frente a seu recurso terapêutico de maior relevo durante o século XX: o medicamento.

A partir do anúncio do isolamento da salicilina, molécula biologicamente ativa do extrato do salgueiro (Salix alba), em 1828, pelo pesquisador alemão Johann Buchner (PIERPOINT, 1997), foi inaugurada uma cosmologia biomédica ocidental, em que o conhecimento tradicional e a planta medicinal são considerados pontos de partida, pertencentes à natureza, que através da tecnologia científica se tornam totalmente desvinculados de seus atributos originais. Para exemplificar esta abordagem, observamos o caso do isolamento da molécula precursora da progesterona sintética - diosgenina - que se tornaria a matéria-prima das pílulas anticoncepcionais. Tal substância foi isolada de algumas espécies do gênero Dioscorea na década de 1950, no México, e a planta de origem, após ser destituída de sua molécula de interesse, perdeu sua utilidade medicamentosa, servindo apenas como referência histórica do medicamento (KLEICHE-DRAY, 2008; MANDY, 2008).

Desta forma, as plantas medicinais como entes botânicos perderam seu significado original no repertório terapêutico da medicina moderna. A botânica medicinal foi abolida como disciplina da formação médica, sendo substituída pela farmacologia das monodrogas representadas pelo denominado "princípio ativo" (FERNANDES, 2004), isto é, moléculas isoladas, biologicamente ativas, 
desvinculadas das espécies botânicas das quais se originaram. As trajetórias históricas foram apagadas, transformando esses medicamentos em artefatos de laboratório, frutos da inovação tecnológica.

Neste sentido, como os médicos prescritores neste início do séculoXXI poderiam ser sensibilizados para aderir à nova terapêutica dos fito-hormônios, uma vez que as plantas medicinais foram historicamente desvalorizadas pela biomedicina como recurso terapêutico legítimo? Uma abordagem analítica de Latour (1983) indicaria que esta adesão se tornaria possível a partir da promoção de uma translação dos laboratórios fitoquímico/farmacológico para o consultório do ginecologista, e a tradução dos novos termos e conceitos desenvolvidos nos laboratórios de pesquisa e desenvolvimento, para sua recepção pelo sistema cognitivo dos médicos, no que diz respeito ao medicamento como recurso terapêutico.

É esse processo de translação que podemos observar, por exemplo, no novo lugar concedido a substâncias naturais como viterscarpina, 27-deoxiacteína, isoflavonas, ácido glicirrético, por exemplo, que foram purificadas (isoladas, identificadas e quantificadas) a partir de plantas medicinais, por intermédio de técnicas de laboratório e equipamentos sofisticados. Essas técnicas têm como objetivo atender a critérios de controle de qualidade do produto farmacêutico, sendo por este motivo chamadas substâncias marcadoras (SCHENKEL et al., 2003).

Tais substâncias, ao contrário de uma planta medicinal, só podem ser percebidas através de processos que Latour denomina de inscrição, pois são detectadas por aparelhos específicos - high performance liquid chromatography (HPLC), ${ }^{8} \mathrm{e}$ computadores, que através de um registro impresso indicam suas presenças e quantidades num extrato vegetal (LODDER, 2009). As substâncias marcadoras, que em alguns extratos são também o princípio ativo da planta, passaram a ser tema prioritário nos materiais de marketing dos laboratórios farmacêuticos dirigidos aos médicos ginecologistas. É factível pensar que em alguns casos, na prescrição de tais substâncias, poderia passar despercebido que a seus pacientes estava sendo administrado o extrato de uma planta, que além de conter essas substâncias consideradas terapêuticas para o climatério, incluem várias outras moléculas que fazem parte da constituição da planta (SCHENKEL et al., 2003). Nessa translação, portanto, o médico não está prescrevendo um princípio ativo isolado - como acontecia com os produtos sintéticos - mas um pool de substâncias, dentre as quais aquela considerada indicada para a terapia hormonal. 
As substâncias marcadoras, portanto, antes importantes somente para químicos e farmacólogos, num ambiente de laboratório científico, passaram a ganhar visibilidade no meio médico. No caso da soja (Glycine max), por exemplo, sua utilização como preventiva nos sintomas associados ao climatério partiu da observação da ligação entre o hábito alimentar nas populações de mulheres de origem japonesa e a baixa frequência de sintomas da menopausa, quando comparadas com mulheres ocidentais (MURKIES et al., 1986). A divulgação desse estudo abriu toda uma linha de pesquisa por parte de vários laboratórios, em busca de um novo medicamento para competir com os hormônios sintéticos. Após o isolamento, identificação e quantificação de um grupo de substâncias que mostraram ter comportamento estrogênio-like, o extrato da soja passou a ser recomendado pelos médicos através do nome referência destas substâncias, a saber, “isoflavonas” da soja (DEF, 2004).

A planta medicinal hierarquicamente passou a ter valor secundário, em detrimento de uma ou mais substâncias nela contidas, tomando-se a parte pelo todo, num típico discurso de orientação mecanicista. Desta forma, segundo Alves \& Silva (2003) - livro escrito por dois médicos ginecologistas da Universidade Federal do Rio de Janeiro voltado para a orientação aos médicos ginecologistas sobre os fito-hormônios - a planta Vitex agnus-castus, utilizada desde a Antiguidade grega, para ser considerada eficaz deverá conter $5 \%$ de viterscarpina e as doses terapêuticas deverão ser de $400 \mathrm{mg} /$ dia de extrato padronizado. As informações terapêuticas do conhecimento tradicional desta planta, depois de sofrer intervenções de cunho científico nos ambientes de laboratórios, passaram a ter suas propriedades terapêuticas traduzidas na linguagem médico-científica, na expectativa de serem assim mais bem compreendidas e absorvidas.

Destaca-se nesse processo a realização de eventos específicos para divulgação científica no meio médico especializado, como o I Simpósio Internacional sobre Fitoterápicos: "Evidências Científicas da Moderna Fitoterapia-Passado, Presente, Futuro", realizado em 6 de abril de 2001, no Hotel Sheraton, Rio de Janeiro, e patrocinado pelo Laboratório Boehringer Ingelheim. Neste evento, durante todo o dia palestraram eminentes pesquisadores do Brasil e da Suíça, apresentando dados sobre a pesquisa em geral de fitoterápicos e do produto a partir da planta Cimicifuga racemosa que viria a ser lançado pelo laboratório patrocinador (ÉPOCA, 2001). O simpósio tinha como público-alvo os principais médicos 
ginecologistas do Brasil e América do Sul, ligados a universidades e hospitais, que foram convidados a participar, sem custos. Segundo a entrevista publicada na revista Época, o diretor de pesquisa do laboratório, Wolfgang Wuttke, afirmou que pesquisas da Universidade de Göttingen, na Alemanha, atestaram os efeitos do remédio natural. "O desempenho é o mesmo do hormônio sintético" (ÉPOCA, 2001). Este produto, no entanto, teve posteriormente seu registro rejeitado pela Agência Nacional de Vigilância Sanitária (ANVISA, 2001) e está fora do mercado brasileiro até o momento atual.

É interessante observar que as abordagens de sensibilização por parte do público médico-científico e consumidor para estas medicaçôes "naturais" explicitam a ideia da oposição entre as categorias "medicamentos sintéticos" e "medicamentos naturais". Na mesma matéria citada acima, havia o depoimento de uma mulher que dizia que com o conhecimento do ginecologista, trocou as pílulas de estrogênio sintético por cápsulas de soja. "Para mim, o melhor médico é Deus. E a natureza, sua farmácia” (ÉPOCA, 2001). Tal depoimento é seguido por muitos outros, que seguem o mesmo tom de oposição entre a medicação sintética e a natural, como na reportagem do Programa "Globo Repórter", veiculada em 2006:

A psicóloga [...] toma os hormônios naturais há quase oito anos, depois de várias tentativas com os hormônios sintéticos. [...] "Cheguei a tentar vários tipos diferentes, dosagens diferentes, [...] e nada funcionava bem. Com os fito-hormônios passou tudo, não sinto mais nada", ela conta. [...] A terapeuta [...] revela: "Nunca me agradou muito tomar hormônio sintético, queria alguma coisa alternativa".

E ainda no site "Saúde Integral", da naturopata Suzete Barreto, onde a maior parte das 714 mulheres que deixaram comentários em reação ao artigo "Reposição Hormonal na Menopausa” (BARRETO, 2007), como pode ser observado em alguns exemplos:

[...] eu não quero ficar tomando estas drogas não, eu quero natural.

Estou no processo de adaptação da menopausa [...]. Tenho os sintomas citados pela maioria dos comentários. Não me adaptei a TRH convencional. Estou procurando uma solução natural para recuperar meu bem estar. Gostaria de saber quais os medicamentos naturais para repor hormônio sem agredir meu organismo.

Hoje já não menstruo mais e também preciso fazer a reposição hormonal, porém prefiro da forma mais natural possível, sem muita agressão ao meu organismo, já tão agredido. 
[...] tirei o útero, comecei a fazer reposição hormonal, o médico receitou o estrogênio conjugado $0,625 \mathrm{mg}$ [faz um ano que troquei com extrofem $1 \mathrm{mg}$ ], me sinto bem mas tenho medo de câncer na mama, pensei em trocar por isoflavona pó natural [...].

A ideia de uma natureza rousseauniana, que tende à perfeição, curadora e segura, pode ser percebida quando há alusão à ausência de efeitos colaterais neste tipo de medicamentos, em contrapartida ao medicamento sintético, produzido pela ciência, por sua vez considerado como artificial e iatrogênico.

No material de marketing do livro Fito-hormônios: abordagem natural da terapia hormonal (ALVES; SILVA, 2003), também há uma evocação de uma noção rousseauniana de natureza, bem como uma reificação da oposição "natural" x "sintético", como segue neste trecho do texto:

[...] é o tipo de trabalho científico que a um só tempo contempla o valor terapêutico das ervas e plantas - neste livro particularizando as açôes hormonais, bem como a minimização de efeitos colaterais; a par de obviamente enfocar a medicação, bem mais barata em comparação com os sucedâneos sintetizados em laboratórios da indústria farmacêutica. (ALVES; SILVA, 2003; 4a capa).

No entanto, quando seguimos para a continuação do texto, percebemos uma abordagem vitalista do conceito, mesclado a informações descritivas da classificação dos reinos biológicos e da botânica científica, como segue:

Revive-se aqui a essência da energia vital da natureza que aviva todos os seres do micro e do macrocrossomos, dos reinos: vegetais, animais, fungos, protistas (microalgas e protozoários) e monera (bactérias e algas azuis). E é no reino vegetal que encontramos o maior número de espécies, muitas ainda não classificadas pelos botânicos. (ALVES; SILVA, 2003, 4a capa).

Ainda nessa obra, nos deparamos com uma linguagem de caráter médicocientífica sobre as plantas medicinais em questão, expressa nas citações de ensaios clínicos e análises de viés farmacológico, e, principalmente, pela utilização dos marcadores fitoquímicos, como a viterscarpina, entendida como um "aproximador" da ideia de princípio ativo. A planta é quase sempre apresentada na sua versão "extrato seco padronizado", numa abordagem similar a tantos outros artigos científicos sobre o tema que vêm sendo veiculados no meio médico-científico sobre plantas medicinais. Neste sentido, a categoria "extrato seco padronizado" pode ser entendida como um quase-objeto híbrido (LATOUR, 1994), pertencente à rede sócio-técnica das plantas medicinais, pois se trata de uma matéria de origem vegetal, que tem como atributo o conhecimento tradicional associado ao qual 
foram agregados saberes e valores científicos-tecnológicos, e que apesar disso ainda é identificado como um "produto natural".

A ideia de uma rede sócio-técnica envolvendo as plantas medicinais foi primeiramente utilizada por Branquinho (2007). Segundo a autora, esta rede tem uma série infinita de possibilidades de articulação de conceitos científicos e não científicos, ligando antigas tradições de conhecimento das plantas às tecnologias avançadas, humanos e não-humanos, natureza e cultura, popular e científico, formando um tecido único, um conjunto de "híbridos" (BRANQUINHO, 2007, p. 19).

No caso dos fito-hormônios, a observação do percurso deste objeto e os nós que marcam os atores envolvidos neste processo, apontam como os médicos ginecologistas, as mulheres menopausadas, chefes de pesquisas de laboratórios farmacêuticos internacionais, o mercado mundial de medicamentos, o setor de terapias alternativas, a Women's Health Initiative, e o "extrato seco padronizado" revelam uma rede que expõe a dissolução dos limites de dentro e de fora do laboratório. As tramas desta rede se traduzem em movimentos de mão dupla, para "dentro" e para "fora" do laboratório.

\section{Considerações finais}

Ao percorrer a trajetória da construção de uma nova categoria terapêutica, podese perceber o fito-hormônio como um fato científico construído, e como os limites do espaço científico são extrapolados, para ganhar outra dimensão nos consultórios de médicos ginecologistas e entre mulheres no período do climatério. Este aspecto do fato científico vem sendo analisado com frequência nos estudos sociais das ciências nos últimos anos, onde as teorias de Bruno Latour, entre outros autores, têm sido centrais para a compreensão de como a construção do saber científico não está necessariamente restrita ao ambiente científico, apontando para fronteiras esvanecidas entre o laboratório e o mundo lá fora.

Neste processo, foi possível localizar o contexto no qual se inseriu o desenvolvimento dos fito-hormônios, e fatores que influenciaram a consolidação desta terapêutica: primeiro, o clima de desconfiança instalado após o anúncio dos riscos associados à TRH; e um ambiente sócio-cultural-econômico específico que teve sua emergência nos anos 1960-70, da revalorização de aspectos ligados à ideia de natureza e consequente alteração nos hábitos de consumo, com ecos consistentes nas décadas posteriores. 
Consideramos que tais eventos foram marcados por controvérsias e que este aspecto, no entanto, não foi abordado no artigo, pois o eixo analítico privilegiado nas análises teve como foco a resignificação da ideia da natureza. O papel da categoria natureza e seu correlato "natural" é central neste episódio, podendo-se observar como a ideia de natureza foi sendo ressignificada ao longo desse processo, segundo a percepção e característica de cada ator pertencente à rede sócio-técnica das plantas medicinais. Se, para as pacientes, a natureza traz um aspecto de segurança em relação ao medicamento, para os cientistas envolvidos neste processo ela é uma fonte de matéria-prima e inspiradora de novos componentes terapêuticos elaborados pela ciência - este sim, com garantia de segurança e eficácia. Já os médicos parecem oscilar entre estas duas visões. O caráter híbrido do extrato seco padronizado, como um artefato constituído de natureza e cultura, parece contemplar a ambiguidade intrínseca aos medicamentos fitoterápicos, neste caso os fito-hormônios, possibilitando a adesão dos médicos prescritores a esta nova terapêutica.

Por outro lado, é interessante observar nos depoimentos das pacientes uma concepção de que os medicamentos naturais seriam isentos de efeitos colaterais, mas buscam a mesma eficácia dos medicamentos sintéticos. Curiosamente, segurança e eficácia são as premissas no desenvolvimento de novos medicamentos pela ciência moderna. Elas são a justificativa de parte da comunidade científica para a não-utilização dos métodos "naturais", pois estes não teriam passado pelas metodologias científicas que garantiriam eficácia e segurança, mesmo que, paradoxalmente, os hormônios sintéticos, aprovados nesses testes, tenham sido questionados pelo próprio campo científico.

\section{Referências}

ALONSO, J. Tratado de Fitomedicina: bases clínicas e farmacológicas. Buenos Aires: ISIS, 1998. ALVES, D.; SILVA, C. Fito-hormônios: abordagem natural da terapia hormonal. São Paulo: Atheneu, 2003. 105p.

AGÊNCIA NACIONAL DE VIGILÂNCIA SANITÁRIA. Instituição - Câmaras Técnicas e Medicamentos - CATEME - Pareceres. Reunião de 7 de agosto de 2001. Disponível em: <http://www.anvisa.gov.br/medicamentos/cateme/parecer/20010807. htm> Acesso em: 10 jul. 2009.

AZIZE, R. Saúde e estilo de vida: divulgação e consumo de medicamentos em classes médias urbanas. In: LEITÃO; D.K.; LIMA, D.N.O.; MACHADO, R.P. (Org.). Antropologia e consumo: diálogos entre Brasil e Argentina. Porto Alegre: AGE, 2006. p. 119-137. 
BARRETO, S. Reposição hormonal na menopausa. 2007. Disponível em: <http://www. saudeintegral.com/artigos/reposicao-hormonal-na-menopausa.html>. Acesso em 02 nov. 2012. BRANQUINHO, F. O poder das ervas na sabedoria popular e no saber cientifico. Rio de Janeiro: Mauad X, 2007. 157p.

CAMPBELL, C. A orientalização do Ocidente: reflexões sobre uma nova teodiceia para um novo milênio. Religiāo e Sociedade. Rio de Janeiro, v. 18, n. 1, p. 5-22, 1997.

. The romantic ethic and the spirit of modern consumerism. London: Alcuin Academics, 2005. 281p.

CAPASSO, F. et al. A. Phytotherapy: a quick reference to herbal medicine. Berlin: Springer, 2003. DICIONÁRIO DE ESPECIALIDADES FARMACÊUTICAS. 2004/2005. Rio de Janeiro: Publ. Científicas; 2004. 929 p.

DUARTE, L.F. et al. Família, reprodução, e ethos religioso: subjetivismo e naturalismo como valores estruturantes. In: DUARTE, L.F.D. et al. (Org.). Família e religiāo. Rio de Janeiro: Contra Capa, 2005. p.15-50.

DUARTE, L. F. A pulsão romântica e as ciências humanas no Ocidente. Revista Brasileira de Ciências Sociais, v. 19, n. 55, p. 5-18, 2004.

ÉPOCA. A saúde brota das plantas: Remédios naturais ganham o aval dos cientistas e alimentam um mercado de US\$ 500 milhões no Brasil. Edição n. 153, de 23 abr 2001. Disponível em: <http://epoca.globo.com/edic/20010423/soci9a.htm>. Acesso em: 10 jul. 2009.

FERNANDES, T.M.D. Plantas Medicinais: memória da ciência no Brasil. 1 ed. Rio de Janeiro: Fiocruz, 2004.

FERREIRA, S. (Org.). Medicamentos a partir de plantas medicinais no Brasil. Rio de Janeiro: Academia Brasileira de Ciências, 1998. 131p.

GAMA, M.P.R. et al. Editorial. WHI: A revelação de uma arriscada promessa da medicina. Endocrinologia \& Diabetes Clínica e Experimental. Curitiba, v. 4, n. 2, p. 213-214, mar 2004. GAEDCKE, F.; STEINHOFF, B. Herbal medicinal products. Stuttgard: Medpharm Scientific, 2003.

GALENA. Trifollin ${ }^{\circledR}$ Red Clover, 2012a. Disponível em: <http://www.galena.com.br/ MagistralBuscaProduto.aspx> Acesso em: 02 nov 2012.

GALENA. Isoflavin Beta. 2012b. Disponível em: <http://www.galena.com.br/ MagistralLinhaProduto.aspx> Acesso em: 02 nov 2012.

IFTODA, D.M. et al. Cimicifuga racemosa L., Trifolium pratense L. e Vitex agnus-castus L.: a correspondência das indicações contida nas bulas dos fitoterápicos e o respaldo científico. Rev. Ciênc. Farm. Básica Apl., v. 27, n. 2, p. 169-176, 2006.

KHANDEKAR, J.; VICTOR, T.; MUKHOPADHYAYA, P. Endometrial Carcinoma Following Estrogen Therapy for Breast Cancer: Report of Three Cases. Arch Intern Med., v. 138 , p. $539-541,1978$. 
KLEICHE-DRAY, M. Institutionalization of Chemistry in Mexico during the twentieth century (1934-1970). In: INTERNATIONAL CONFERENCE ON THE HISTORY OF CHEMISTRY, "NEIGHBORS AND TERRITORIES. THE EVOLVING IDENTITY OF CHEMISTRY”, 6. Proceedings... 2008. p. 739-749.

KOLATA, G.; PETERSEN, M. The New York Times. Hormone Replacement Study: A shock to the Medical System. 2002. Disponível em: <http://www.nytimes.com/2002/07/10/us/ hormone-replacement-study-a-shock-to-the-medical-system.html? pagewanted $=$ print $>$. Acesso em: 12 jul 2009.

LAINE, C. Postmenopausal hormone replacement therapy: how could we have been so wrong? Ann Intern Med., v. 20, n. 137, p. 290, Aug 2002.

LATOUR, B. Jamais fomos modernos. Rio de Janeiro: Ed. 34, 1994. 150p.

Give Me a Laboratory and I Will Raise the World. In: KNORR-CETINA, K.D.; MULKAY, M. Science Observed. London: Sage, 1983. p. 141-170.

LATOUR, B.; WOOLGAR, S. A vida de laboratório: a produção dos fatos científicos. Rio de Janeiro, Relume Dumará, 1997. 310p.

LENOBLE, R. História da ideia de natureza. Lisboa: Ed. 70, 1990. 367p.

LIPSETT, M. B. Estrogen use and cancer risk. JAMA, v. 237, p. 1.112-1.115, 1977.

LODDER, R. University of Kentuch, Advanced Science and Technology Center. History of HPLC. 2009. Disponível em: <http://kerouac.pharm.uky.edu/asrg/hplc/history.html>. Acesso em: 16 jul 2009.

LUZ, M. Novos saberes e práticas em saúde coletiva. 3 ed. São Paulo: Hucitec, 2007. 172p.

MANDY, R. Yams of fortune: the (uncontrolled) birth of oral contraceptives. Journal of Young Investigators, v. 6, issue 7, s/p, 2003. Diponível em: <http://www.jyi.org/features/ ft.php?id=540>. Acesso em: 24 abr 2010.

MARTINS, A. Novos paradigmas e saúde. Physis: Revista de Saúde Coletiva, Rio de Janeiro, v. 9, n. 1, p. 83-112, 1999.

MURKIES, A.l.; WILCOX, G.; DAVIS, S. Clinical review 92: Phytoestrogens. The Journal of clinical endocrinology and metabolism, v. 83, n. 2, p. 297-303, 1998.

NEWALL, C.; ANDERSON, L.; PHILLIPSON, J. Herbal medicines: a guide for health care profissionals. London: The Pharmaceutical Press, 1996.

NOGUEIRA, M.I.; CAMARGO JR, K.R. A orientalização do Ocidente como superfície de emergência de novos paradigmas em saúde. História, Ciências, Saúde-Manguinhos. Rio de Janeiro, v. 14, n. 3, p. 841-861, jul.-set. 2007.

OBSERVATÓRIO DA IMPRENSA. Reposição Hormonal. Diferentes formas de apresentar uma polêmica, 2002. Disponível em: <http://www.observatoriodaimprensa.com.br/ofjor/ ofc310720023p.htm>. Acesso em: 13 abr 2010. 
OUDSHOORN, N. United we stand: the pharmaceutical industry, laboratory, and clinic in the development of sex hormones into scientific drugs, 1920-194 Science, Technology, \& Human Values, v. 18, n. 1, p. 5-24, 1993.

On the making of sex hormones: research materials and the production of knowledge. Social Studies of Science, v. 20, p. 5-33, 1990.

PIERPOINT, W. Edward Stone (1702-1768) and Edmund Stone (1700-1768): confused identities resolved. Notes Rec. R. Soc. Lond. London, v. 51, n. 2, p. 211-217, 1997.

ROHDEN, F. O império dos hormônios e a construção da diferença entre os sexos. História, Ciências, Saúde - Manguinhos. Rio de Janeiro, v. 15, supl., p. 133-152, 2008.

SAYD, J.D. Novos paradigmas e saúde: notas de leitura. Physis: Revista de Saúde Coletiva, Rio de Janeiro, v. 9, n. 1, p. 113-121, 1999.

SCHENKEL, E. et al. Produtos de origem vegetal e o desenvolvimento de medicamentos. In: SIMÕES, C. et al. (Org.). Famacognosia: da planta ao medicamento. Porto Alegre: UFRGS, 2003. p. 372-400.

SMITH, D.C. et al. Association of exogenous estrogen and endometrial carcinoma. $N$ Engl J Med., v. 4, n. 293, p. 1.164-7, Dec 1975. Disponível em: <http://content.nejm.org/cgi/ content/abstract/293/23/1164> Acesso em: jun 2009.

SONAGLIO, D. et al. Desenvolvimento tecnológico e produção de fitoterápicos. In: SIMŌES, C. et al. (Org.). Famacognosia: da planta ao medicamento. Porto Alegre: UFRGS, 2003. p. 289-326.

STRATHERN, M. After Nature: English kinship in the late twentieth century. Cambridge: Cambridge University Press, 1992. 240p.

SCHULTZ, V.; HANSEL, R.; TYLER, E. Fitoterapia racional: um guia de fitoterapia para as ciências da saúde. 4 ed. Barueri: Manole, 2002.

WAGNER, H.; WISENAUER, M. Fitoterapia: fitofármacos, farmacologia e aplicações clínicas. 2.ed. São Paulo: Pharmabooks, 2006.

WEISS, N. Noncontraceptive estrogens and abnormalities of endometrial proliferation. Ann Intern Med., v. 88, p. 410-412, 1978.

WEISS, N. et al. Endometrial cancer in relation to patterns of menopausal estrogen use. JAMA, v. 242, p. 261-264, 1979. Disponível em: <http://www.revistaendocrino.com/page7. html>. Acesso em: jul 2006.

WOMEN'S HEALTH INITIATIVE INVESTIGATORS. Risks and benefits of estrogen plus progestin in healthy postmenopausal women. JAMA, v. 288, p. 321-333, 2002. Disponível em: <http://www.whiscience.org/>. Acesso em: jun 2009.

VEIGA, A.; SEGATTO, C. Traídas pela medicina: um golpe na confiança. Revista Época. 2002. Disponível em: <http://revistaepoca.globo.com/Epoca/0,6993,EPT341671-1880,00. html> Acesso em: 13 abr 2010. 
VIEIRA, C.S.; NAVARRO, P.A.A.S. Síndrome climatérica. Revista Brasileira de Medicina, v. 64, n. 12, p. 99-109, dez 2007. Disponível em: <http://www.moreirajr.com.br/revistas. asp? fase $=$ r003\&id_materia $=3688>$. Acesso em: 13 abr 2010.

VIGETA, S. A experiência da perimenopausa e pós-menopausa com mulheres que fazem uso ou não da terapia de reposição hormonal, 2004. Disponível em: <http://200.130.7.5/spmu/ portal_pr/mulher_ciencia/redacoes_2006/PG_Vigeta_SP.pdf> Acesso em: fev 2007.

ZIEL, H.K.; FINKLE, W.D. Increased risk of endometrial carcinoma among users of conjugated estrogens. $N$ Engl J Med., v. 4, n. 293, p. 1.167-70, Dec 1975. Disponível em: $<$ http://content.nejm.org/cgi/content/short/293/23/1167?query=nextarrow $>$. Acesso em: jun. 2009.

\section{Notas}

${ }^{1} \mathrm{O}$ termo fito-hormônio foi o primeiro a ser utilizado pela mídia, permanecendo como o mais conhecido. No entanto, outros nomes foram atribuídos posteriormente a esta categoria de medicamentos, como fitoestrógenos, empregado por alguns pesquisadores.

${ }^{2}$ Substâncias de origem vegetal que agem como precursoras de hormônios femininos e masculinos (progesterona, estrogênio e testosterona), ou que promovem açôes clínicas/farmacológicas semelhantes aos hormônios endógenos.

${ }^{3}$ As inscrições são o resultado da transformação da matéria, mediada através de toda uma sorte de equipamentos de registro, em dados escritos que serão utilizados pelos pesquisadores na elaboração de um artigo (Cf. LATOUR; WOOLGAR, 1997, p. 44).

${ }^{4}$ Este trabalho é uma versão ampliada e reestruturada analiticamente de trabalho originalmente apresentado no GT25 (Natureza, Corpo, Sentidos) da 26a. Reunião Brasileira de Antropologia, realizada de 01 a 04 de junho de 2008 em Porto Seguro, Bahia, Brasil. Agradeço a Ana Teresa A. Venâncio pela revisão do manuscrito destinada a esta publicação.

${ }^{5}$ Orientalização é um termo utilizado por Campbell no sentido de uma incorporação de paradigmas próprios das culturas ocidentais, como alguns sistemas religiosos como o hinduísmo e budismo, práticas alimentares e de atividade física e de saúde que, no entanto, não afetaram significativamente a essência (natureza básica) do sistema cultural Ocidental (CAMPBELL, 1997).

${ }^{6}$ Campbell utiliza a noção de teodiceia, nos termos de Weber, como a explicação dos caminhos de Deus para o homem, e especialmente a solução do "problema do mal" (CAMPBELL, 1997, p. 5).

${ }^{7}$ A este respeito, conferir os trabalhos de Sayd (1999), Martins (1999) e Nogueira \& Camargo Jr (2007).

${ }^{8}$ HPLC é a abreviação de high performance liquid chromatography, nome do equipamento desenvolvido nas décadas de 1970-80, que possibilitou separar, identificar, purificar e quantificar os vários componentes de um extrato vegetal, emitindo relatórios impressos sobre a amostra analisada (cf. LODDER, 2009). 


\begin{abstract}
"Phyto-hormones": Science and nature in the treatment of menopause

A new product called "phyto-hormone" was designed to replace the hormone replacement therapy (HRT) in recent years. This development is associated with two historical contexts: the announcement of the risks associated with hormone replacement therapy (HRT) for postmenopausal women in 2002, and a change in public perception of consumers and pharmaceutical companies about medicinal plants. Some plants used by traditional communities for symptoms associated with menopause have been scientifically researched in recent decades and, as a result, a new category of medicine was established. From the actor-network theory and translation, Bruno Latour analyzes the construction of this class of drug, and how the idea of "natural" was re-signified by the actors involved in the socio-technical network.
\end{abstract}

> Key words: menopause; HRT; medicinal plants; nature. 